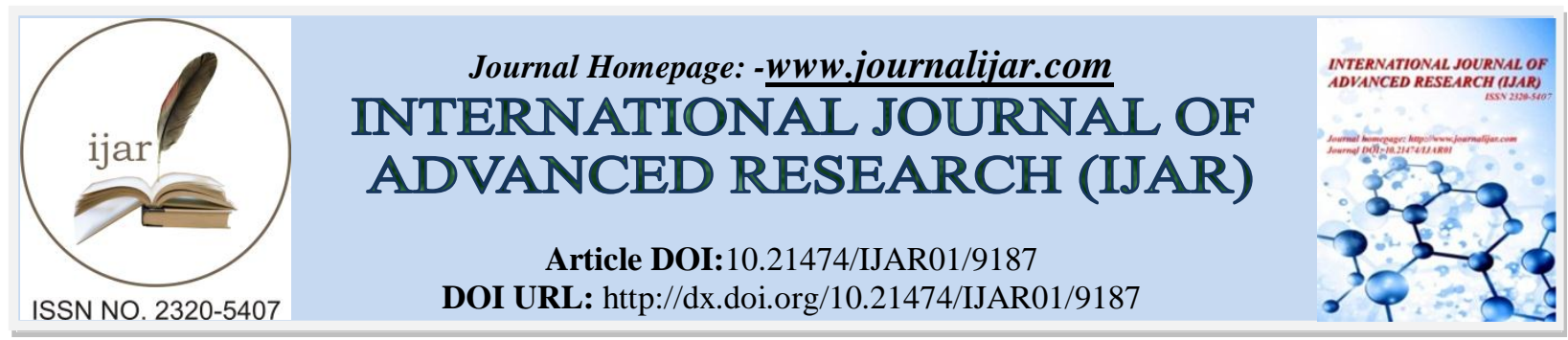

RESEARCH ARTICLE

\title{
APPLICATION OF HEALTH BELIEF MODEL ON FACTORS CONTRIBUTING TO RELAPSE, FAILURE AND LOSS TO FOLLOW UP IN TUBERCULOSIS PATIENTS.
}

Puneet Kaur, Sukhpal Kaur,Amarjeet Singh and Sandhya Ghai.

\section{Manuscript Info}

\section{Manuscript History}

Received: 02 April 2019

Final Accepted: 04 May 2019

Published: June 2019

\section{Key words:-}

Tuberculosis, Loss to follow up, Relapse, Treatment failure, Care givers, DOTS providers, Health Belief Model.
Abstract

Background: There are numerous public health and clinical consequences of incomplete TB treatment. Treatment with second-line drugs is less successful and more toxic. Relapse and treatment failure are also important problems because patients can harbor drug resistant mycobacterium tuberculosis bacilli.

Objective: To explore the patients' perspective about factors associated with loss to follow up, relapse and treatment failure in tuberculosis patients based on concepts of Health Belief Model.

Materials and Methods: This phenomenological study was conducted on 12 patients registered at DOTS centers, U.T. Chandigarh. Purposive sampling technique was used for data collection. A pre validated semi structured interview guide was used to collect the data. In-depth interviews were conducted. Audio recording was done following permission from the participants. Principle of redundancy was followed for data collection.

Results: Based on patients' verbatim 12 themes were formulated. These were Factors contributing to relapse; Factors contributing to loss to follow up; Factors contributing to relapse; Physical problems related to disease conditions; Emotional stress; Stigma related to disease; Economic challenges; Job related challenges; Family support; Health care system related challenges; Attitude of DOTS Providers; Expectations of National Programme. Each theme was further divided in to various sub-themes to categorize the information more appropriately.

Conclusion: This study has demonstrated many factors responsible for loss to follow up, treatment failure and relapse in TB patients based on Health Belief Model. This model was really beneficial and provided framework to explore the patients perspective related to loss to follow up, relapse and treatment failure in tuberculosis patients. It is concluded that although medicines were provided free of cost but side effects of medicines and pills burden were disabling factor in completion of treatment. Lack of adequate food, side-effects, pill burden of the drugs, stigma and discrimination were factors that contributed to poor TB treatment adherence in the study area.In addition to this low socioeconomic status, family liabilities, awareness issues and burden of losing income from work contributed to non-compliance. Addictions, imbalanced diet, primary resistance, secondary infection and non compliance to treatment contributed to treatment failure and relapse of 
disease. Patients were knowledge deficit related to spread and treatment of tuberculosis.

Copy Right, IJAR, 2019,. All rights reserved.

\section{Introduction:-}

The theoretical and conceptual framework explain the path of a research and ground it firmly in theoretical constructs. Conceptual foundations of professional nursing practice comprehensively explore issues and concepts that influence professional practice and the delivery of nursing care. The overall aim of the conceptual framework is to make research findings more meaningful, acceptable to the theoretical constructs in the research field and ensures generalizability. ${ }^{1}$

Tuberculosis is a communicable, lethal disease (if not treated) is influenced by biological, environmental, psychological and social factors. ${ }^{2}$ The health belief model is a good tool for nurses, offering them a theoretical framework for helping their patients to prevent chronic disease like tuberculosis or, if disease is present, improve quality of life. The health belief model is a way of encouraging patients to realize their susceptibility to health conditions with the goal of inspiring positive changes. ${ }^{3}$

Lack of adherence to health-promoting advice challenges the successful prevention and management of tuberculosis. There are numerous public health and clinical consequences of incomplete TB treatment. The patients develop resistance to first line ATT drugs. Treatment with second-line drugs are less successful and more toxic. Relapse and treatment failure are also an important problem because patients can harbour drug resistant mycobacterium tuberculosis bacilli.Patients' poor adherence to anti-TB therapy, with an estimate of as low as $40 \%$ in developing countries, remains the principal cause of treatment failure globally. ${ }^{4}$

The WHO recommends at least $85 \%$ cure rate of all diagnosed TB cases. ${ }^{5}$ In order to achieve this cure rate, adherence needs to be in the order of $85-90 \%$. $^{6}$ Lack of access to formal health services, traditional beliefs leading to self-treatment, loss of income, lack of social support, drug side effects, pill burden, lack of food, stigma with lack of disclosure, and lack of adequate communication with health professionals are some of the factors that can lead to loss to follow up in patients.

Risk factors for relapse included drug irregularity, initial drug resistance, smoking, and alcoholism, shorter total duration of treatment (particularly Rifampicin), poor adherence during treatment (mainly during intensive phase), use of fewer than three drugs in intensive phase, greater disease severity and cavitations, high bacterial load, smoking, being male, the presence of concomitant disease, being underweight, and infection with HIV. ${ }^{8}$

There are many reasons for treatment failure in patients receiving appropriate regimens. These include: Nonadherence, drug resistance, malabsorption of drugs, laboratory error and a few patients take a long time to respond as part of extreme biological variation. ${ }^{9}$

\section{Health Belief Model}

The Health Belief Model (HBM) is a psychological model that attempts to explain and predict health behaviors. This is done by focusing on the attitudes and beliefs of individuals. The HBM was first developed in the 1950s by social psychologists Hochbaum, Rosenstock and Kegels working in the U.S. Public Health Services. The model was developed in response to the failure of a free tuberculosis (TB) health screening program. This model addresses how a person perceives a threat of health problem and what behaviour he is adapting for managing the problem. The health belief model asserts that when a person believes he or she is susceptible to a health problem with severe consequences, the person will more likely conclude that the benefits outweigh the barriers associated with changing one's behaviour to prevent the problem.

This model explain that people will adopt recommended behaviour only when perceived threat of disease and benefits of action will outweigh their perceived barriers to action ${ }^{10}$. 


\section{Core Assumptions and Statements}

The HBM is based on the understanding that a person will take a health-related action (i.e., Anti-tubercular drugs) if that person:

1. feels that a negative health condition (i.e., Disseminated Tuberculosis) can be avoided,

2. has a positive expectation that by taking a recommended action, he/she will avoid a negative health condition (i.e. ATT will cure Tuberculosis), and

3. believes that he/she can successfully take a recommended health action (i.e., he/she can use ATT comfortably and with confidence).

\section{The Major Concepts of Health Belief Model are: Modifying variables:}

It consists of Individual characteristics, including demographic, psychosocial, and structural variables which can affect perceptions of health-related behaviors.

Demographic variables include person's age, sex, race, ethnicity, and education. Psychosocial variables include personality, social class, and peer and group pressure. Structural variables include knowledge about a given disease and prior contact with the disease, among other factors. The health belief model suggests that modifying variables affect health-related behaviours indirectly by affecting perceived seriousness, susceptibility, benefits, and barriers. In present study those factors is explored by taking in-depth interviews of participants. Patients who are educated, young and independent understand the counselling and have capability of comprehending things. It increases their chances of adherence to treatment. At times their personality also reflects their course of treatment. Patients who have carefree attitude tend to forget medicines as compared to patients who are serious related to completion of treatment.

\section{Perceived Susceptibility:}

Perceived Susceptibility refers to subjective assessment of risk of developing a health problem due to default, relapse or re-occurrence of tuberculosis.

Individuals who feel that they are at risk of developing particular health problem generally engage in behaviours to reduce their risk of developing the health problem. On contrary individuals who deny that they are at risk developing TB infection don't go for screening and health check-up. In present study perceived susceptibility was explored by exploring their feelings related to their susceptibility of developing infection.

\section{Perceived Severity:}

Perceived Severity refers to subjective assessment of the severity of a health problem and its potential consequences. Individuals who perceive a given health problem as serious are more likely to engage in behaviours to reduce its severity. For instance if patient believe that TB is a fatal disease then he will follow strict compliance with the treatment. He will go for follow up, investigations and complete their treatment without default. Perceived severity was explored by exploring their feelings related severity of tubercular infection and its consequences.

\section{Perceived seriousness:}

Perceived seriousness explains about persons beliefs about the disease itself (e.g., whether it is life threatening or may cause disability or pain) as well as impacts of the disease on his functioning in work and society.

For instance, an individual may perceive that there would be serious financial consequences as a result of being absent from work for several days, then he or she may perceive tuberculosis to be a particularly serious condition. Patient's perceived severity in the current study was explored by exploring their exploring his views regarding tuberculosis.

\section{Perceived Benefit:}

Perceived Benefits refer to an individual's assessment of the value or efficacy of engaging in a health promoting behaviour to decrease risk of disease.

If an individual believes that a particular action will reduce susceptibility to a health problem or decrease its seriousness, then he or she is likely to engage in that behaviour regardless of objective facts regarding the effectiveness of the action. For example, individuals who believe that ATT will help them in curing tuberculosis are 
more likely to comply to the treatment. In the current study perceived benefit was explored by exploring their feelings related to benefits in adherence to anti-tubercular medicines.

\section{Perceived Barrier:}

Perceived barriers refer to an individual's assessment of the obstacles to behaviour change.

Even if an individual perceives a health condition as threatening and believes that a particular action will effectively reduce the threat, barriers may prevent engagement in the health-promoting behaviour. In other words, the perceived benefits must outweigh the perceived barriers in order for behaviour change to occur. Perceived barriers to taking action include the perceived inconvenience, expense, danger (e.g. side effects of a medicine) and discomfort (e.g., pain, emotional upset) involved in engaging in the behaviour. For instance, side effects of anti-tubercular medicines may act as barriers to receiving the ATT. In the current study perceived barrier was explored by exploring their feelings related to physical, social, economical and psychological effects of taking anti-tubercular drugs.

\section{Cues to Action:}

It explain that a cue or trigger is necessary for prompting engagement in health-promoting behaviours. It can be internal or external. Physiological Cues (e.g. pain, symptoms) are an example of internal cues to action. External Cues include events or information from close others i.e. the media, health care providers and motivation from care givers promote engagement in health-related behaviours.

Cues to Action such as symptoms of tuberculosis, print material, pill calendar, text messages, television, radio, social media can be use to instigate compliance to tuberculosis treatment. Role of family members also plays important role in providing psychological support and reducing stigma among patients suffering from tuberculosis. Perceptions of care givers and DOTS providers were explored in this study. Care givers perception and attitude towards the disease was explored. DOTS providers' challenges and strategies to help TB patients was ruled out.

\section{Self-efficacy:}

Self-Efficacy refers to an individual's perception of his or her competence to successfully perform a behaviour.

People are more likely to adopt a health behaviour if they think they will be successful. For instance if patient feel that he will be able to complete the treatment and will get over the tuberculosis infection, he will comply with the ATT drug. In the current study self-efficacy was explored by discussing their feelings related to their ability and capability of completing tuberculosis treatment.

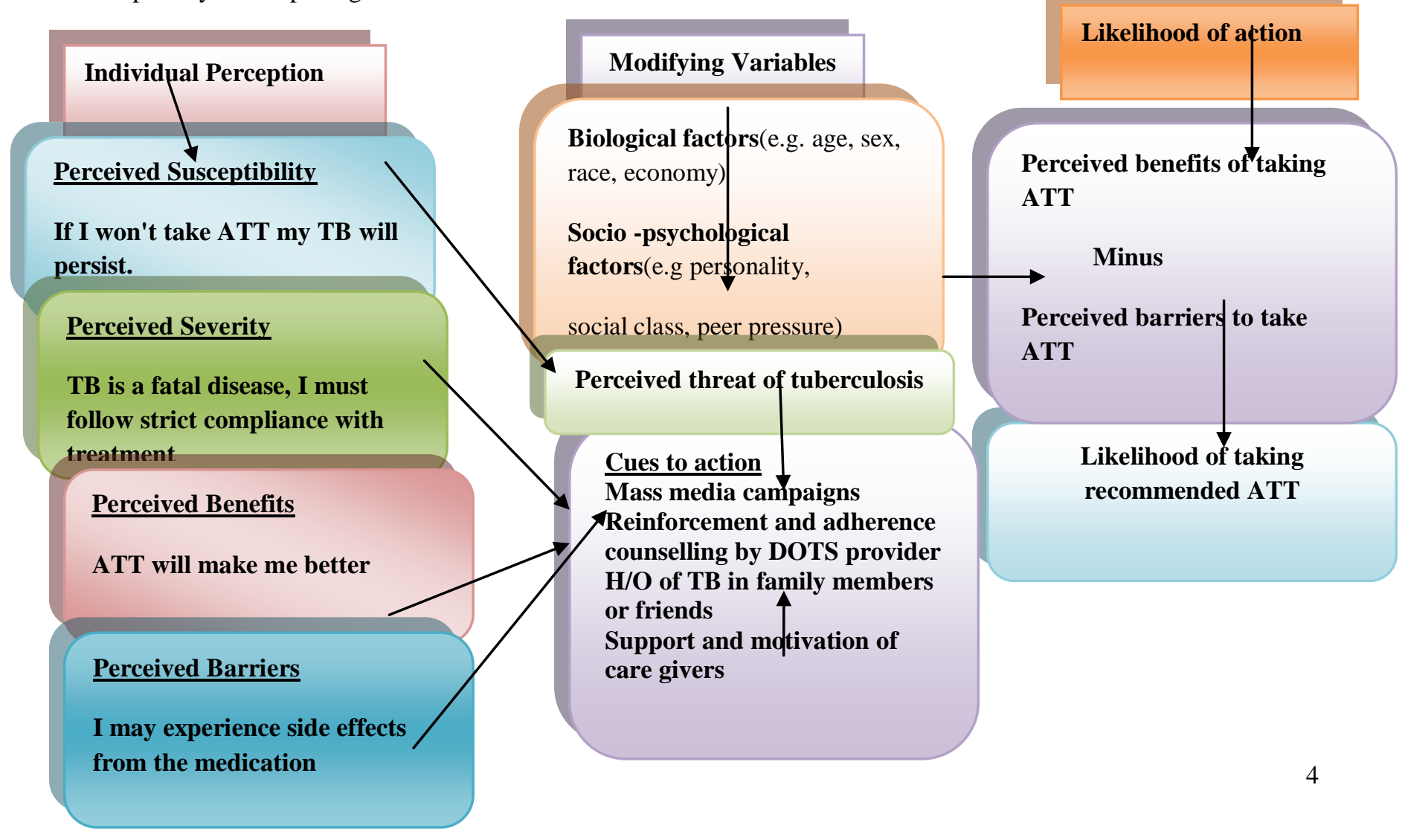


Figure 1:-Conceptual framework based on Health Belief Model

\section{Objective:}

To explore the patients' perspective about factors associated with loss to follow up, relapse and treatment failure in tuberculosis patients registered at U.T. Chandigarh.

\section{Material and Methods:-}

1. Qualitative research approach was used in the study, because it is based on the realities, experiences and view points of the tuberculosis patients. Phenomenological research design is selected to explore the lived experiences of 12 patients who were loss to follow up, relapse and treatment failure.

2. This study was conducted at DOTS Centres in various health settings in U.T Chandigarh. Eight DOTS Centres were selected from 4 tuberculosis units, based on number of patients registered and population size to cover major part of U.T Chandigarh. Quarterly reports of patients census were collected from State TB officer, Sec 34 and two DOTS Centres from each TB unit were selected for data collection to cover U.T. Chandigarh. The research study was conducted in the months of July -Sept 2017.

3. Purposive Sampling technique was used to draw samples for the present study. A pre validated semi structured interview guide was used to collect the data. In-depth interviews were conducted. Audio recording was done following permission from the participants. Principle of redundancy was followed for data collection. Patients who were not traceable and who had at extensive dyspnoea were excluded from the study.

The tools used for data collection were developed through extensive literature search, consultation with experts in the field of pulmonary medicine, public health and nursing education and researcher's experience in the field of tuberculosis. Tools used for the study were interview schedule consisting of socio demographic data sheet clinical profile and semi structured in-depth interview guide for patients.

Data collection was done at the DOTS centres UT, Chandigarh. Patients were interviewed on Monday, Wednesday, Friday i.e. on DOTS day between 8am-2pm as they come to DOTS centres for their medicines. Quiet and separated room was selected at DOTS Centres to carry out in-depth interviews. Subjects were made comfortable and rapport was developed by asking warm up questions i.e. about their well being and personal information. Interviews were recorded by using audio tape recorder. On an average, the time spent for each interviews varied from 25-30 minutes, according to the willingness of the client to talk.

Ethical clearance for the study was obtained from the Institute Ethical Committee, PGIMER, Chandigarh and state TB Officer, RNTCP, Chandigarh. An informed written consent was obtained from the study subjects after explaining the objectives and duration of their involvement.

\section{Results:-}

Based on patients' verbatims, 12 themes were formulated. These were Factors contributing to relapse; Factors contributing to loss to follow up; Factors contributing to relapse; Physical problems related to disease conditions; Emotional stress; Stigma related to disease; Economic challenges; Job related challenges; Family support; Health care system related challenges; Attitude of DOTS Providers; Expectations of National Programme. Each theme was further divided in to various sub-themes to categorize the information more appropriately.

\section{Discussion:-}

The present study has been one of the very few research endeavours to identify factors associated with loss to follow up, relapse and treatment failure as per TB patients' perspective using Health Belief Model. Researcher wanted to gain insight into their thoughts, feelings and problems, so phenomenology design was selected as a medium to enter into their private world. The study identified the various factors which are responsible for loss to follow up, relapse and treatment failure according to patients.

\section{Factors affecting compliance to TB treatment Medicines related factors}

The treatment non-compliance is recognized as one of the major challenges in achieving TB control. Medicines related issues like side effects of medicines, pill burden were responsible for non-adherence to treatment. In India traditional medicine system is deeply rooted. Patients perceive that traditional system of medicine is more beneficial 
and have less side effects as compared to ATT. Patients generally have notion that allopathic medicines are hot in nature and will make them sicker. Patients usually go to traditional healers and take ayurvedic medicines when they feel sick. This perceived behaviour acts as a barrier in receiving ATT. One of patient reported "I could not take TB medicines as I was not able to swallow them. Medicines are very thick; they cause acidity and irritation in my stomach. I couldn't tolerate the suffocation and ghabrahat because of medicines, so I left the treatment in between." Other patient had apparent relief reported that "My fever was down and cough was relieved, appetite was also improved. I missed medicines once or twice and I did not take my medicines. I did not go to take medicines." On the contrary some patients left treatment in between as they didn't feel improvement in their condition."After taking medicines my condition got worse. Earlier I had no fever but I used to get high grade fever after taking medicines. I was getting sicker that's why I left the treatment in between."

Similar findings were shown in study a by Kaona ${ }^{11}$ which revealed that major factors leading to non-compliance included patients beginning to feel better $(45.1 \%$ and $38.6 \%)$, lack of knowledge on the benefits of completing a course $(25.7 \%)$, running out of drugs at home (25.4\%) and TB drugs being too strong $(20.1 \%$ and $20.2 \%)$.

Study by Boru in Ethopia (2014) revealed that many patients were unable to adhere to their treatment because of one or a combination of the following factors; lack of adequate food, poor communication between healthcare providers and patients, beliefs in traditional healing system, unavailability of the service in nearby health facilities, side-effect and pill burden of the drugs, stigma and discrimination. The patients take their anti-TB medications under difficult circumstances and experienced a wide range of interacting factors. ${ }^{12}$

\section{Disease related factors}

Physical problems related to disease contributed to non compliance to treatment. Patients had social role limitations due to physical problems, bodily pain, and decreased vitality. Women had burden of house care, child care and employment which was obstacle in gaining access to diagnostic facility and completing their adequate treatment. Patients feel that they are not self -efficient in taking treatment of Tuberculosis. One of patient responded "My body has become loose. I feel weak. I don't feel like working, if family members are not at home then I can't go to DOTS Centre for treatment alone. Due to disease condition patients feel weak and if they don't have support system in family then they don't travel to DOTS centre for their treatment.

Similar findings were reported in a study by Guo ${ }^{13}$ which showed that quality of life aspects affected by TB included physical functioning and emotional/mental well-being.

\section{Finance related factors}

Tuberculosis affects the most productive age group and the resultant economic cost for the society is high. This disease has considerable impact on patient's household's in terms of income, health, education and nutrition, particularly if patient is a wage earner. Socio-economic factors were also important contributory factors to noncompliance to treatment. The financial constraints that recognized through lack of money, unemployment due to disease, transportation cost, food has been continued to exert their influence on TB patients. One patient stated "We really have dirth of money. I used to work as a sweeper at Sec 32 hospital, now I can't work because of disease. My wife is working as a sweeper in PGI .One daughter is studying in school. I have one son of $22 \mathrm{yrs}$ old, he doesn't work. It is very difficult to earn living for us."

A study conducted by Rajeshwari ${ }^{14}$ revealed that the total costs, and particularly indirect costs due to TB, were relatively high. The average period of loss of wages was 3 months. Care giving activities of female patients decreased significantly.

\section{Awareness Issues}

Lack of knowledge and misconceptions about transmission of disease lead to discrimination like separate utensils for food or drink. Diagnosis of TB is associated with increase anxiety/tension, fear of loss of wage/earning, and stigma threatening self-esteem and quality of life. Present study showed that there was knowledge deficit in patients and care givers related to disease and course of treatment. "I just know that TB is a disease in which we have to take precautionary measures. It can be fatal if it spreads. I don't know about how this disease occurs and spreads." Patients' and care givers' were not aware about the factors responsible for causation and transmission of TB infection. "TB spreads by virus. It spreads if we talk to patients. We should not go near TB patients." There was knowledge gap related to the course and duration of treatment. They also did not have information about the need 
and importance of adherence of medicines in patients. This calls for cue to action by DOTS providers by providing information, promote awareness and reminders to patients.

Study by Chinnakali revealed that out of 395 participants, 370 (94\%) respondents had heard about TB. Regarding the symptoms of TB, $82 \%$ were aware that cough is a symptom of TB. Among the $79 \%$ of study subjects who reported any test to diagnose TB, sputum examination as a method of diagnosis was known to only $40 \%$. However, $84 \%$ of the subjects were aware of the free treatment available for TB under National program. ${ }^{15}$

\section{Stigma related to disease}

Despite being curable, tuberculosis is still a stigmatized disease. Not only is TB patients' suffering due to its clinical manifestations, but also because of society's prejudice, embarrassing situations, and even self-discrimination. This study revealed that stigma related to disease was very much prevalent in society. Patients were kept isolated from other family members. One patient reported "I stay in isolation. My room is separate. I don't sit and lie down with anyone in summers and winters. I don't eat with anyone. My utensils and clothes are separate from other family members."

Patients usually avoided participation in social activity because of stigma related to TB " I really feel bad when there is some marriage in my family, they don't call me for any function. They stay away from me as if I am having Leprosy. I really don't like it, then my husband counsel and console me that you don't worry, you will be fine."Patients' had feeling of worthlessness and hopelessness due to disease condition.

Patients also reported that they didn't disclose their TB status at office as they feared of losing their job because of their TB status. "I feel scared that because of TB I may lose my office friends. They may get drift away from me. I fear I may lose my job as well."

Female patients had a fear of not getting married because of the disease. They were worried about their future due to stigma and discrimination related to disease. "Our society has hatred towards tuberculosis. I am tensed that I will get married or not. I get proposals but it doesn't get accepted. This is the reason I have started the treatment all over again. I am taking care of my diet as well."

Study conducted by Liefooghe revealed that due to fear patients often denied the diagnosis and rejected the treatment. While both male and female TB patients faced many social and economical problems, female patients were more affected. Divorce and broken engagements seemed to occur more often in female patients. Females were usually economically dependent on their husbands and family in law, and needed their cooperation to avail of treatment. ${ }^{16}$

Study by Dias found that TB still causes patients to suffer from fear of transmission, social prejudice, and death. Despite the fact that the emotional support provided by families and healthcare professionals is considered essential to treatment adherence and completion, participants in this study reveal that friends and colleagues have distanced themselves from them for fear of contagion and/or prejudice. ${ }^{17}$

\section{Health care related factors}

Health care workers' attitude, clinic opening times, availability of medicines, accessibility issues were important factors contributing for treatment compliance. All the respondents in the study revealed that attitude of health workers was friendly except one who reported that attitude of health worker was unprofessional and unfriendly. This represent the support patients receive from National Health Programme.

One of the respondents in the study reported that no counselling was given to her related to the treatment and no follow up was planned for her when she was on treatment. She completed 6 months treatment in 4 months as complete box was given to her without any counselling and DOTS was not initiated."I have taken complete treatment but I finished my course in 4 months because proper information was not given to me regarding duration of treatment. I finished the course in 4 months instead of 6 months. When I went to return the box they told me not to disclose it to anyone, they said I could have died as I took double doses. They did not run any test nor they came home or called me to DOTS Centre." 
Study conducted by Bonsu defined service satisfaction as involving education/counselling (on drugs, nature of condition, sputum production, caregivers and contacts of patients), patient follow-up, assignment of reliable treatment supporters as well as being attentive and receptive to patients, service availability (e.g. punctuality at work, availability of commodities), positive assurances about disease prognosis and respect for patients. ${ }^{18}$

\section{Factors contributing for relapse and treatment failure}

Smoking, drinking alcohol were the important factors related to relapse and treatment failure. The implications of patients taking alcohol while on treatment are threefold. Firstly, patients may forget to take their medicines under influence of alcohol, secondly there may be more side effects of TB medicines particularly when patients are taking alcohol with treatment. Thirdly there are chances of relapse of disease if patient continues to take alcohol after completion of treatment. "I got relapse of TB due to my carelessness, I used to drink daily when I was fine and used to do smoking as well."

"The reason behind relapse of TB is that, I did not leave drinking. I used to drink on non DOTS day. I continued to drink after my recovery. They told me to leave drink but I continued the same without informing them. I made this mistake. "

Exposure to occupational hazards may also have contributed to relapse of disease. Patients reported that job profile and working conditions were responsible for relapse of disease. "At my work place there is lot of dust, when we do paper cutting there is lot of dust in surroundings. I get bad cough because of this, I do get dyspnoea as well. This may be the reason behind relapse of tuberculosis. "Other patient said "It may be due to my job profile, there is lot of dust due to coal when we iron clothes. I get dyspnoea because of coal."

A study conducted by Mlotshwa et al revealed that male gender, HIV co-infection and a $>2+$ acid fast bacilli (AFB) smear grading at the start of TB treatment were independent risk factors for non-conversion $(\mathrm{p}<0.001)$. Age was a risk factor for non-conversion in new cases, but not for re-treatment cases. ${ }^{19}$

A study by Morsy et al revealed that significant risk factors for treatment failure were non-compliance to treatment, deficient health education to the patient, poor patient knowledge regarding the disease and diabetes mellitus as comorbid condition. ${ }^{20}$

Lack of balanced diet and non adherence to medicines were also reported by patients to contribute to relapse of disease. Patients were not able to eat balanced diet due to socio- economic factors or side effects of medicines like anorexia.One patient reported "I don't eat proper diet this may be the reason behind relapse of tuberculosis. I can't eat proper diet. Sometimes I don't feel like eating anything."

Other patient responded "The reason for relapse is I did not follow the due course of medicines, instead of finishing the course in 6 months I finished in 4 months. I finished the course of medicines much earlier than the due course. After that I did not get myself checked nor did I undergo any test."

Primary resistance to medicines was a major factor of treatment failure. If a patient is already resistant to antitubercular medicines then he will not respond to the current regimen available for TB. Many patients take treatment from private practitioner, traditional healers, quacks which make them resistant to medicines.

\section{Expectations from National Programme}

There were several responses from patients when they were asked about their opinion on what could be done to help TB patients. Patients had many expectations from health programme. Majority of patients wanted reduction in pills so that they can take medicines easily and it would decrease side effects of medicines. "There should be change in medicines, medicines are very heavy and it is difficult for patients to take them. If they can decrease the quantity of medicines then it will be easy for patients to take them."

In this study patient who had to travel a distance to get treatment wanted his treatment to be started at nearest DOTS Centre. He responded "I am taking medicines what they are giving me, but I have to travel quite a lot for treatment. No one comes to take medicine by their choice they come when they are unwell. If a person is having fever then only he will rush to doctors. Everyone wants to take treatment from nearby health facility. I wish they start my treatment from nearest health facility." 
Patients wanted government should take more initiative to spread awareness about the disease. They reported that they were not aware about the disease, its mode of spread and treatment. "Government should give correct and clear information regarding TB so that patients develop clear understanding related to disease. Half knowledge is always dangerous."

The patient responses highlighted the need to address financial and nutritional support for those who can't afford diet and were unemployed due to disease condition. They appreciated that govt. is doing good by providing free medicines but also emphasized the need for balanced diet with medicines. "If govt. can provide financial support then, we will be able to buy good diet. Government is doing good by providing free medicines as TB treatment and tests are expensive, but diet is also important with medicines."

\section{Conclusion:-}

This study has demonstrated many factors responsible for loss to follow up, treatment failure and relapse in TB patients based on Health Belief Model. This model was really beneficial and provided framework to explore the patients perspective related to loss to follow up, relapse and treatment failure in tuberculosis patients. It is concluded that although medicines were provided free of cost but side effects of medicines and pills burden were disabling factor in completion of treatment. Lack of adequate food, side-effects, pill burden of the drugs, stigma and discrimination were factors that contributed to poor TB treatment adherence in the study area.In addition to this low socio-economic status, family liabilities, awareness issues and burden of losing income from work contributed to non-compliance. Addictions, imbalanced diet, primary resistance, secondary infection and non compliance to treatment contributed to treatment failure and relapse of disease.Patients were knowledge deficit related to spread and treatment of tuberculosis.

\section{Refernces:-}

1. Creasia JL, Parkar B. Conceptual foundations of proffessional nursing practice. St Louis: Mosby, Year Book; 1991.

2. Smeltzer C Suzanne, Bare G Brenda, Hinkle L Jainice, Cheever H Kerry. Brunner and Suddharth textbook of Medical-Surgical Nursing. 13 ${ }^{\text {th }}$ Edition. New Delhi: Published by Dorling Kindersley (India) Pvt, Ltd; 2014. P.554-65.

3. Marriner TA, Raile AM. Nursing theorists and their work.5th ed.St Louis: Mosby; 2005

4. Ginsberg A, Spigelman M. Challenges in tuberculosis drug research and development. Nature Medicine. 2007;13(3):290-94.

5. World Health Organization. Global tuberculosis control; WHO report 2009. Epidemiology, Strategy and Financing. Geneva, Switzerland; 2009. Available from; WWW.WHO/HTM/TB/2009.411.

6. Fox W. Compliance of patients and physicians experience and lessons from tuberculosis-II. BMJ.1983;287(6385):101-5.

7. Heemanshu A, Satwanti K. Determinants of lost to follow up during treatment among tuberculosis patients in Delhi. International Journal of Medical Research \& Health Sciences 2016; 5, 1:145-52.

8. BHIVA - 12.0 Management of relapse, treatment failure and drug resistance [Internet]. Bhiva.org. 2018 [cited 22 March 2017]. Available from: http://www.bhiva.org/120Managementofrelapsetre.aspx

9. Cox HS, Morrow M, Deutschmann PW. Long term efficacy of DOTS regimens for tuberculosis: Systematic review. Br Med J. 2008;336:484-7.

10. Health Belief Model [Internet]. Utwente.nl. 2017 [cited 20 January 2017].Available from: https://www.utwente.nl/cw/theorieenoverzicht/Theory\%20Clusters/Health\%20Communication

11. Kaona F, Tuba M, Siziya S, Sikaona L. An assessment of factors contributing to treatment adherence and knowledge of TB transmission among patients on TB treatment. BMC Public Health. 2004; 4(1):92-97

12. Gugssa Boru C, Shimels T, Bilal A. Factors contributing to non-adherence with treatment among TB patients in Sodo Woreda, Gurage Zone, Southern Ethiopia: A qualitative study. Journal of Infection and Public Health. 2017;10(5):527-33.

13. Guo N, Marra F, Marra C. Measuring health-related quality of life in tuberculosis: a systematic review. Health and Quality of Life Outcomes. 2009;7(1):14.

14. Rajeswari R, Balasubramanian R, Muniyandi M, Geetharamani S, Thresa X, Venkatesan P. Socio-economic impact of tuberculosis on patients and family in India. International Journel of Tuberculosis and Lung diseases. 1999 Oct;3(10):869-77.

15. Chinnakali P, Gurumurthy J, Ramakrishnan J, Vasudevan K, Upadhyay R, Panigrahi K. Level of awareness 
about tuberculosis in urban slums: Implications for advocacy and communication strategy planning in the National program. Lung India. 2013;30(2):139

16. Liefooghe R, Michiels N, Habib S, Moran M, De Muynck A. Perception and social consequences of tuberculosis: A focus group study of tuberculosis patients in Sialkot, Pakistan. Social Science \& Medicine. 1995; 41(12):1685-92.

17. Dias A, Oliveira D, Turato E, Figueiredo R. Life experiences of patients who have completed tuberculosis treatment: a qualitative investigation in southeast Brazil. BMC Public Health. 2013;13(1).250-55

18. Bonsu F, Afutu F, Hanson-Nortey N, Ahiabu M, Amo-Adjei J. Satisfaction of tuberculosis patients with health services in Ghana. International Journal of Health Care Quality Assurance. 2017; 30(6):545-53

19. Mlotshwa M, Abraham N, Beery M, Williams S, Smit S, Reddy C et al (2016). Risk factors for tuberculosis smear non-conversion in Eden district, Western Cape, South Africa. BMC Public Health. 2016;16:365

20. M Morsy, HH Zaher, MH Hassan (2003). Predictors of treatment failure among tuberculosis patient sunder DOTS strategy in Egypt. Eastern Mediterranean Health Journal.2003;9(4): 180-87. 